\title{
Assessment of paediatric head shape and management of craniosynostosis
}

Kimberley Kai Lun, Shivani Aggarwala, Danielle Gardner, Jeremy Hunt, Erica Jacobson, Raj Reddy, Mark Gianoutsos, Michael Rtshiladze

\section{Background}

The presentation of a child with an abnormal head shape can be challenging and should be met with an appropriate clinical approach. Craniosynostosis is a common cause of paediatric skull deformity and is best managed by a multispecialty tertiary referral unit with regular follow-up. As craniosynostosis frequently requires time-sensitive surgery, it is important to differentiate between craniosynostosis and common selflimiting conditions such as deformational plagiocephaly.

\section{Objective}

The aim of this article is to outline the clinical approach to paediatric skull deformity in the general practice setting, and to highlight the importance of early referral if there is clinical suspicion of craniosynostosis.

\section{Discussion}

Parental concern regarding infant head shape is common. General practitioners (GPs) have an important role in assessment, diagnosis and referral for paediatric skull deformities. GPs are well placed to clinically differentiate between deformational plagiocephaly and craniosynostosis and provide timely referrals to optimise patient outcomes.
THERE IS A WIDE SPECTRUM of normal head shapes, and parental concern regarding the shape of their infant's head is common. Newborn head shapes are varied and influenced by intrinsic factors such as parental head shape, sex and ethnicity, and external factors including fetal moulding, prematurity, prolonged labour and instrumented deliveries. Consequently, newborn head shapes are challenging to assess accurately.

Parents with concerns regarding their child's head shape often first present to general practitioners (GPs). GPs play an important part in providing first-line assessment, reassurance and referral if craniosynostosis is suspected to allow time-sensitive treatment options to be considered.

\section{Craniosynostosis}

Craniosynostosis is the most common paediatric skull deformity requiring specialist craniofacial intervention and often initially presents as an abnormal head shape. Craniosynostosis is the premature fusion of one or more cranial sutures, which causes skull growth restriction in the plane perpendicular to the involved suture, with compensatory overgrowth parallel planes. ${ }^{1}$ This results in distinctive head shapes (Figure 1), which can help determine the involved suture(s) and lead to a diagnosis. ${ }^{2}$

Occurring in one in 2000-2500 live births, ${ }^{1,2}$ craniosynostosis is a heterogeneous condition classified as either non-syndromic (arising as an isolated issue in an otherwise healthy infant) or syndromic (occurring with multisystem extracranial anomalies).

Non-syndromic craniosynostosis arises without extracranial dysmorphisms. It can involve one or more sutures and can present with a wide range of head shapes depending on the sutures affected and the extent of fusion. ${ }^{3}$ The most common presentation is when only a single suture, most commonly the sagittal suture, is involved. ${ }^{4}$ The cause of craniosynostosis is incompletely understood, but the condition is believed to occur early in gestation. The pathogenesis may involve developmental errors during embryogenesis, intrauterine skull compression, genetic factors and/or teratogens. ${ }^{2,3}$

Syndromic craniosynostosis is rarer and accounts for between $9.9 \%$ and $18.3 \%$ of all craniosynostosis cases. ${ }^{5}$ It frequently involves multiple sutures, and children present with a wide range of affected extracranial organ systems. There are over 200 known craniosynostosis-associated syndromes including Muenke, Apert and Crouzon syndrome. ${ }^{6}$

\section{Deformational plagiocephaly}

Deformational plagiocephaly is the most common cause of abnormal head shape and an important differential to consider in all infants with head 
shape abnormalities. Deformational plagiocephaly arises as a result of repeated external pressure to an infant's skull for an extended time period before or after birth, ${ }^{7,8}$ for example, sleeping supine without altering position.

Some craniosynostosis forms, notably unilateral lambdoid synostosis, may produce a similar head shape to deformational plagiocephaly, and differentiating between them can be challenging. ${ }^{9,10}$ Deformational plagiocephaly is self-limiting, and the head shape begins to improve as the child grows and spends less time lying down. Surgery is almost never indicated for deformational plagiocephaly, so differentiating it from craniosynostosis is very important.

Conservative management for deformational plagiocephaly consists of counter-positioning and physiotherapy. Counter-positioning measures include increasing tummy time and moving the cot or placing a night light so that the infant is encouraged to look in the direction opposite to their neck turning. Torticollis may also be associated with deformational plagiocephaly. Torticollis is a condition
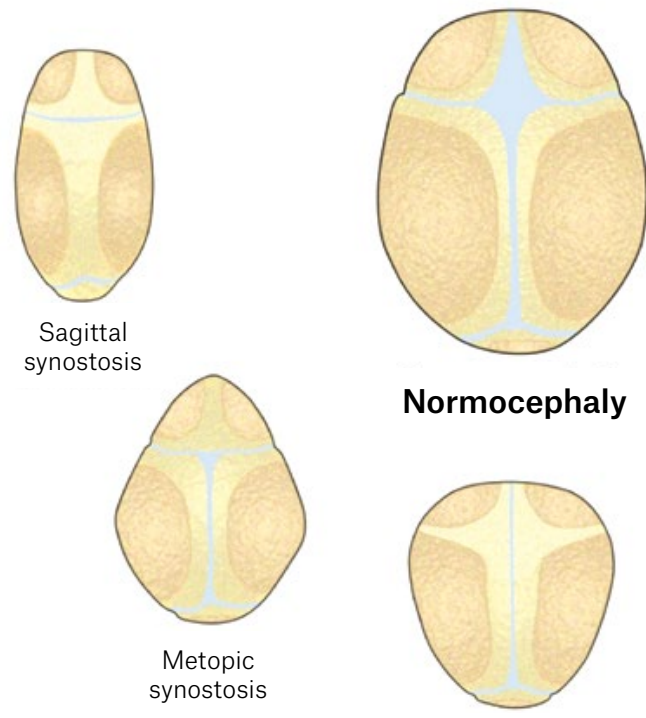

Normocephaly

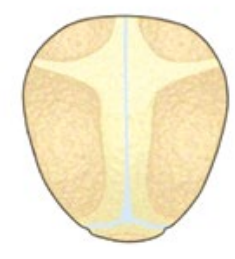

Bilateral coronal synostosis

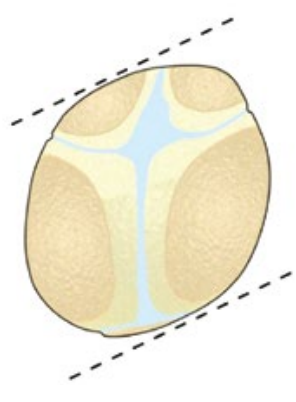

Deformational posterior plagiocephaly

Figure 1. The various distinctive head shapes in the axial plane showing types of craniosynostosis, and deformational plagiocephaly

in which the sternocleidomastoid muscle is tight resulting in an abnormal neck posture. This can cause the child to have a head tilt or turn preference. Torticollis can lead to occipital flattening on the opposite side of the head to the neck stiffness. If the GP finds neck tightness suggestive of torticollis, referral to neonatal or paediatric physiotherapy can provide stretches that may be effective (Box 1).

\section{Clinical approach to a child with an abnormal head shape}

The vast majority of cases of single-sutural craniosynostosis can be diagnosed by clinical examination alone. Expert clinician assessment is a sensitive, accurate and low-risk diagnostic method. ${ }^{9,11}$

GPs have an important role in the initial assessment of a child with an abnormal head shape. The purpose of the initial clinical assessment is to differentiate infants who clearly have deformational plagiocephaly and those who may be presenting with craniosynostosis.

\section{Box 1. Conservative strategies for the management of deformational plagiocephaly}

- Encourage supervised tummy time.

- Alternate the baby's head position during day sleeps; it is important to note that the use of pillows in the cot is not recommended as this is not SIDS safe.

- If bottle feeding, alternate the preferred feeding arm that the baby lies in, so that the baby must turn their head more towards the bottle.

- Turn the pram so that the child is facing forward towards the world to enhance visual and audio stimulation. This will encourage more active head turning.

- Use a low-back highchair and move the highchair position to encourage the baby to turn to look around.

- Encourage carrying or sitting the baby out of the pram/capsule to relieve external pressure on the head.

- Refer to a paediatric physiotherapist if there is evidence of torticollis. Physiotherapists can also help with teaching positioning techniques to parents.

SIDS, sudden infant death syndrome 
A thorough history from the parents should include when the head shape was first noted to be different, changes in head shape over time, prenatal history and developmental history including motor development to elicit factors that may contribute to decreased exposure to prone positioning. A careful clinical examination of head shape is critical, as overall shape is important in determining the diagnosis. Measurement of head circumference should be conducted with the use of paediatric growth charts to track serial measurements over time. It is important to note that the crossing of centiles is more informative than isolated measurements. Patients should also be examined for torticollis by including neck turning in the detailed history from parents and assessing active range of motion of the neck by having the child turn their head each way to look at an object. The key differences between the two conditions are outlined in Tables 1, 2 and 3.
If deformational plagiocephaly is diagnosed, GPs should inform parents, reassure them that the condition is self-limiting and provide strategies to avoid further external pressure (Box 1). Deformational plagiocephaly may be challenging to diagnose because of its wide spectrum of presentations. A guide to the clinical approach in the general practice setting is shown in Figure 2, while presentations that should prompt suspicion of craniosynostosis are outlined in Table 1.

Table 1. Comparison of deformational plagiocephaly and craniosynostosis

\begin{tabular}{|c|c|c|}
\hline & Deformational plagiocephaly & Craniosynostosis \\
\hline Cause & $\begin{array}{l}\text { External pressure to skull } \\
\text { - Back sleeping } \\
\text { - Restricted intrauterine environment } \\
\text { - Muscular torticollis } \\
\text { - Prematurity }\end{array}$ & Premature fusion of cranial suture(s) \\
\hline Risk factors & $\begin{array}{l}\text { Developmental delay (eg hypotonia) } \\
\text { Torticollis } \\
\text { Hip spica cast } \\
\text { Sleeping supine }\end{array}$ & Family history in some cases \\
\hline Ear displacement & Present, anteriorly & Present, towards the affected suture \\
\hline Frontal bossing & Ipsilateral & Contralateral \\
\hline Bulging mastoid & Absent & Present in lambdoid craniosynostosis \\
\hline Palpable ridge & Absent & May be present \\
\hline Torticollis & May be present & Not related/no association \\
\hline Head circumference & Normal rate of head growth & May have slowing of head growth \\
\hline \multirow[t]{2}{*}{ Natural history } & Worsens over the first four months & Appears to remain stable in many cases \\
\hline & $\begin{array}{l}\text { Improves as the child becomes mobile and child's neck } \\
\text { tone improves (eg once the child learns to roll, sit) }\end{array}$ & Will not improve with time \\
\hline
\end{tabular}


If craniosynostosis cannot be excluded or head shape is severely affected, a prompt referral to a multidisciplinary paediatric craniofacial team at a tertiary centre is required. Severe cases of deformational plagiocephaly may benefit from moulding helmet therapy, and referral to a specific helmet service may be warranted. The use of helmet therapy for the treatment of deformational plagiocephaly remains contentious, and proof of their clinical benefit is not universally accepted. ${ }^{12}$

\section{Measurements}

Anthropometric measurements form part of the physical examination. The two measurements most often reported are the head circumference and cephalic index.

Head circumference is measured with a flexible, non-stretchable measuring tape. With the child positioned appropriately, the tape is placed around the head at points just above the eyebrows, above the ears and around the occipital prominence at the back of the head. The tape is pulled to compress the hair, and the measurement is recorded to the nearest $0.1 \mathrm{~cm}$.

Cephalic index measurement requires spreading calipers, making it challenging and unlikely in a general practice setting.
Cephalic index $=$ (Maximum head width $/$ maximum head length) $\times 100$

Moreover, while cephalic index and head circumference may have some applications as a diagnostic and outcome measure, high intra-observer and interobserver variability is a significant problem. Further natural variation of the craniofacial norm has resulted in debate over the value of cephalic index as a meaningful measurement. ${ }^{13}$

\section{Referral}

Craniosynostosis is a complex condition and is managed at a multispecialty tertiary referral unit. The multidisciplinary

\section{Table 2. Three-dimensional photogrammetry images of the various types of craniosynostosis}

\begin{tabular}{|c|c|c|c|c|}
\hline & Front & Side & Back & Top \\
\hline $\begin{array}{l}\text { Sagittal } \\
\text { synostosis }\end{array}$ & & & & \\
\hline $\begin{array}{l}\text { Metopic } \\
\text { synostosis }\end{array}$ & & & & \\
\hline $\begin{array}{l}\text { Unicoronal } \\
\text { synostosis }\end{array}$ & & & & \\
\hline $\begin{array}{l}\text { Unilateral } \\
\text { lambdoid } \\
\text { synostosis }\end{array}$ & & & & \\
\hline
\end{tabular}


team includes a diverse and large group of clinicians. Among them are plastic surgeons, neurosurgeons, ophthalmologists, paediatricians, dental specialists, geneticists, nurses, speech therapists, neuropsychologists and social workers. ${ }^{1,14}$ Regular follow-up throughout childhood is necessary to monitor for head growth, development and the late onset of occult raised intracranial pressure (ICP).
GPs have a key role in referring patients to an appropriate craniofacial unit. While referral is appropriate at any age, early referral ensures maximal treatment options are available. ${ }^{2}$

Table 3. Three-dimensional reconstructed computed tomography scans of the various types of craniosynostosis and deformational plagiocephaly

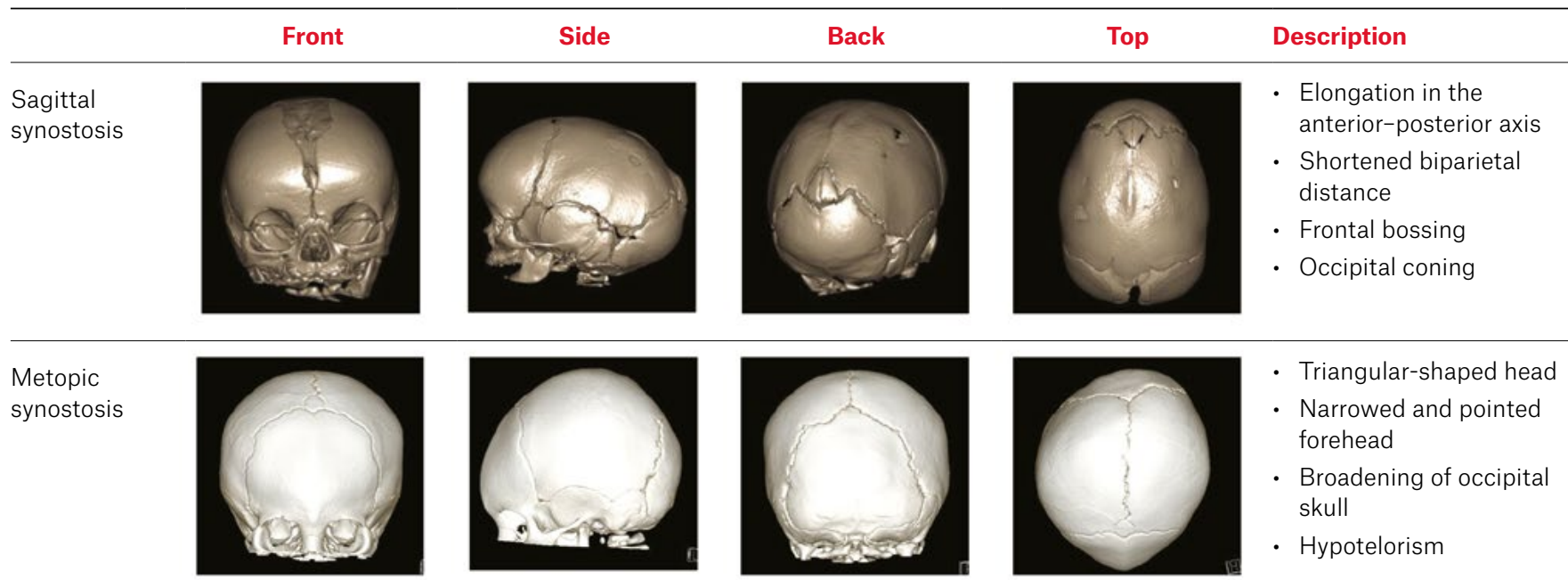

Unicoronal synostosis
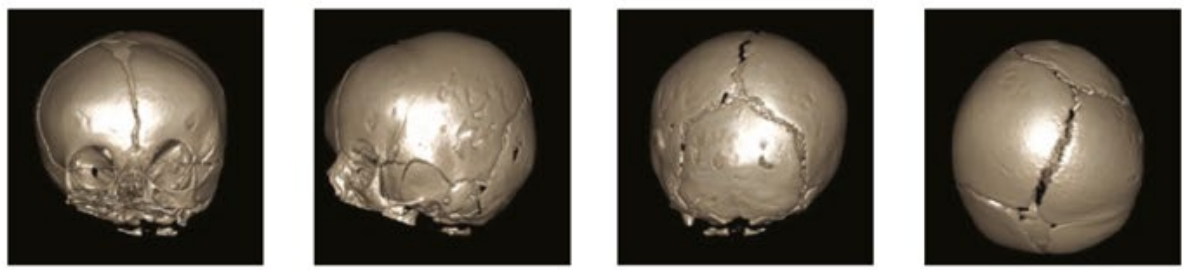

- Forehead flattened on affected side

- Forehead more projected on the unaffected side

- Asymmetry in appearance of eyes (one eye more 'open')

- Deviation of root of nose towards affected side

Unilateral lambdoid synostosis
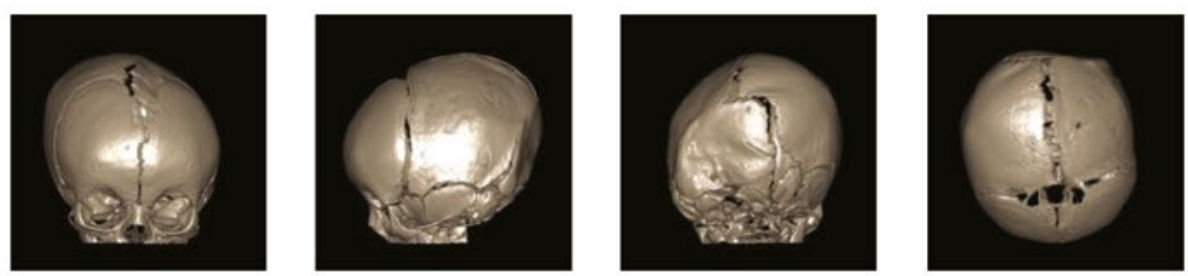

- Occipital flattening and asymmetry

- Ipsilateral ear and mastoid displaced downward

- Head shape from above may resemble a trapezoid

- Asymmetry of cranial base

Deformational plagiocephaly
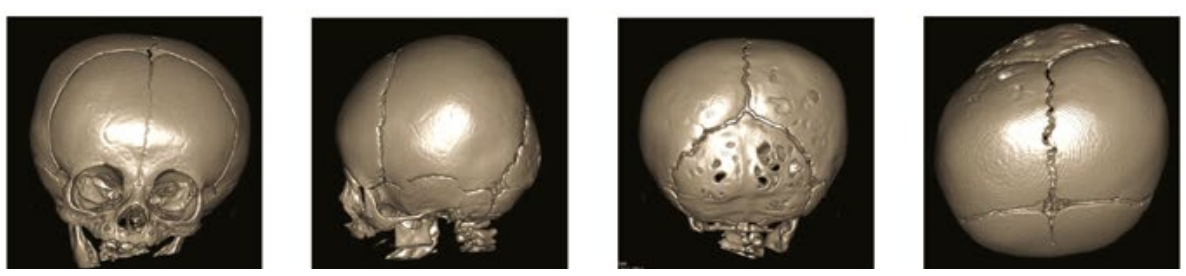

- Parallelogram shape of head

- Ipsilateral ear and forehead displaced anteriorly

- Ipsilateral occipital flattening accompanied by contralateral occipital bossing 


\section{History}

- Was unusual head shape present at birth?

- Has head shape changed or remained stable?

- Has it worsened or improved?

- Prenatal history

- Family history of similar conditions

- Developmental milestones

- Any other identifiable anomalies

\section{Examination}

- Observe head shape from top down, sides, front and back

- Feel for anterior fontanelle - present or absent? Small or large? Soft or firm?

- Feel sutures - is there a ridge or is it flat? Metopic ridge? Overriding or visible sutures?

- Signs of syndromes involving craniosynostosis

(eg syndactyl [fused digits], dysmophic facial features)

\section{Deformational plagiocephaly}

- A parallelogram-shaped head when viewed from the top down

- Present, soft fontanelles

- No obvious ridging or visible sutures

- Anterior displacement of ipsilateral ear

- Head tilt

- May coincide with torticollis

- Contralateral facial flattening

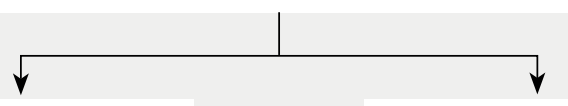

\section{Craniosynostosis}

- An abnormal head shape that coincides with the shapes in Figure 1

- Vary widely depending on which suture is involved

- Absent, firm fontanelles

- Overriding or visible sutures with obvious ridging; may have metopic ridge

- Signs of syndromes involving craniosynostosis (eg syndactyl, dysmorphic facial features)

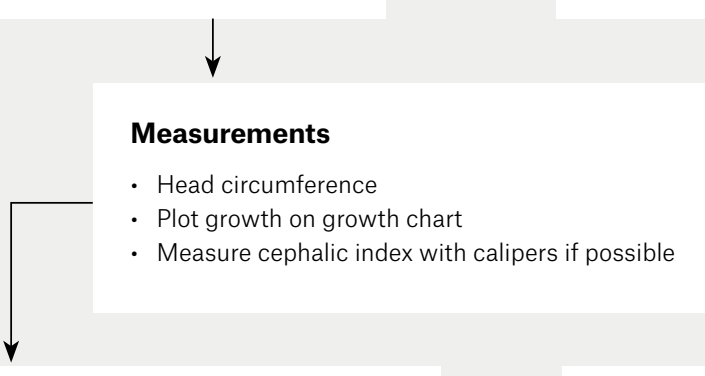

\section{Confirm the cause is likely deformational} plagiocephaly

\section{$\downarrow$}

- Review again within 4-6 weeks

- Discuss conservative measures to reduce external pressure

- Physio referral if torticollis is present

- Consider referral to a helmet therapy service if head shape is severe

\section{Unable to exclude craniosynostosis}

\section{$\downarrow$}

- Refer to a multidisciplinary team by 8-10 weeks of age to ensure patient has maximal options for intervention if needed

Figure 2. Flowchart for clinical approach for general practitioners 


\section{Imaging}

While imaging modalities such as ultrasonography, X-ray and computed tomography (CT) are available, there is no evidence they provide additional diagnostic or treatment benefit. Instead, they may result in unnecessary radiation exposure, sedation risk and financial cost. ${ }^{15}$ Studies have found more than two-thirds of craniofacial surgeons in the USA routinely obtain imaging for every case. ${ }^{16}$ However, in many other units, imaging is only indicated in challenging cases without an obvious clinical diagnosis. ${ }^{11}$ In these cases, a low-dose non-contrast CT scan is the diagnostic imaging of choice.

In cases of suspected craniosynostosis, it is recommended that GPs contact the referral centre prior to organising imaging to avoid unnecessary radiation exposure and referral delay.

\section{Treatment}

\section{Reasons for treatment}

Surgical intervention for craniosynostosis aims to return the cranium to a shape within normal range and reduce the risk of raised ICP.

While uncorrected craniosynostosis tends not to lead to worsening deformity, the abnormal cranial form does not improve with growth or time. ${ }^{17}$ This carries an immense psychosocial burden on patients, especially during emotional development in adolescence..$^{2,18,19}$ Doumit et al found skull deformity was the reason for treatment in $63 \%$ of patients, while raised ICP was the cause for $33 \%$ of patients, and mental disability the cause for $4 \%$ of patients. ${ }^{20}$ Treatments that normalise overall appearance improve the psychosocial domains of social interaction, peer support networks and psychiatric disease frequency. ${ }^{10}$

The association between raised ICP and craniosynostosis remains contested. While the exact rate of raised ICP in craniosynostosis is unknown, estimates suggest a range from $4.5 \%$ to $24 \%$ without treatment. ${ }^{21}$ The cause of raised ICP in craniosynostosis is incompletely understood. Potential causes include growth rate differences between fused skull and underlying brain, blood flow changes, cerebrospinal fluid flow anomalies and brain parenchymal differences. ${ }^{22}$ In syndromic cases, raised ICP can be exacerbated by obstructive sleep apnoea because of the severe midface hypoplasia often present in these conditions. ${ }^{23}$

While the effect of surgery in reducing raised ICP risk is unknown, it is generally accepted that surgery reduces the overall risk. Despite this, a minority of patients may later develop raised ICP, which can, if untreated, lead to optic nerve atrophy, failure to thrive and associations with neurodevelopmental delay. ${ }^{10,24}$ Therefore, all patients with craniosynostosis should continue regular follow-up with a multidisciplinary team into early adulthood.

\section{Treatment approaches}

There is a wide range of surgical approaches for craniosynostosis, with no consensus on an optimal method. ${ }^{20}$

Surgical approaches generally can be divided into early, more limited procedures and later, more extensive remodelling procedures. Notably, in the past decade, less invasive procedures have become increasingly popular. ${ }^{3}$ More limited approaches such as strip craniectomy or spring-mediated cranioplasty are useful in patients under four months of age. ${ }^{25}$ These approaches often require thin, malleable calvarial bone with a maximal potential for growth and for the re-ossification of post-operative bone defects. ${ }^{3}$ For older patients, larger, more comprehensive operations including total cranial vault remodelling and frontoorbital advancement are available. ${ }^{25}$ These procedures are often deferred until later ages to allow for lower anaesthetic risks and a larger circulating blood volume. Still, there is no consensus on the ideal timing of total vault remodelling procedures.

\section{Treatment timing}

Optimal treatment time and the ideal operative window is debatable. Surgery before 12 months of age can reduce potential impacts on crucial brain development, raised ICP risk and skull base deformities. ${ }^{10,26}$ Bone is also easily remodelled, and infants are able to ossify craniectomy defects that occur with surgery. However, there is a larger anaesthetic risk and risk of haemorrhage. Conversely, at 12 months of age or older, bone becomes mineralised and difficult to remodel, yet more stabilised and requires less future growth prediction and potential surgical overcorrection. ${ }^{25}$

Consequently, surgical timing depends on patient factors, such as age and bone maturity, ${ }^{25}$ and informed opinion, with most surgeries performed between three and 12 months of age. ${ }^{3}$ These variations mean different specialist units may have vastly different treatment protocols. Early referral is essential to allow specialist centres the most flexibility and choice with treatment options.

\section{Future diagnostic directions}

Three-dimensional photogrammetry is a recent, reliable, non-invasive measurement that can accurately quantify head volumes to objectify clinical treatment outcomes. ${ }^{27}$ Unlike conventional radiography, it can be conveniently performed in the clinic with no radiation or anaesthetic risk, and the results are comparable to CT in severe craniosynostosis and overall appearance assessment. ${ }^{28}$ It is especially effective in very young infants because of their minimal hair and thin scalp skin, allowing closer visualisation of the skull shape. Complex three-dimensional photogrammetry has become commonplace in many craniofacial units.

The accuracy of three-dimensional photogrammetry lessens with age as children develop more hair and thicker skin. There is also no visualisation of cranial vault and brain, impairing its usefulness in assessing intracranial pathology. ${ }^{28,29}$

Exciting future advances such as machine-learning applications have the potential to be able to aid in the diagnosis of craniosynostosis, especially when combined with three-dimensional photography. Breakthroughs in camera technologies and computer processing are resulting in the miniaturisation of the equipment required to produce three-dimensional photography. Perhaps in the future user-friendly phone 
applications will deliver three-dimensional photogrammetry to a wide range of health professionals and become routine in general practice, ${ }^{27}$ making craniosynostosis identification easier and more reliable. ${ }^{30}$

\section{Key points}

- Parents presenting with concerns regarding their child's head shape are common.

- Deformational plagiocephaly is a common presentation and must be distinguished clinically from craniosynostosis.

- Suspicion that a child is presenting with craniosynostosis warrants urgent referral to a specialist multidisciplinary paediatric service, as surgical interventions are age specific.

\section{Authors}

Kimberley Kai Lun BMedSc, MD, Sydney Children's Hospital, Randwick, NSW

Shivani Aggarwala MBBS, BOptom, MS (Plast), Plastic and Reconstructive Surgery Trainee, Sydney Children's Hospital, Randwick, NSW

Danielle Gardner BN, MPH, Clinical Nurse Consultant - Cleft and Craniofacial, Department of Plastic Surgery, Sydney Children's Hospital, Randwick, NSW

Jeremy Hunt MBBS, FRACS, Visiting Medical Officer, Department of Plastic Surgery, Sydney Children's Hospital, Randwick, NSW

Erica Jacobson MBBS, PhD, FRACS, Head of Department, Department of Neurosurgery, Sydney Children's Hospital, Randwick, NSW

Raj Reddy MBBS, MS, FRACS, Visiting Medical Officer, Department of Neurosurgery, Sydney Children's Hospital, Randwick, NSW

Mark Gianoutsos MBBS, MD, FRACS, Head of Department, Department of Plastic Surgery, Sydney Children's Hospital, Randwick, NSW

Michael Rtshiladze MBBS, BSc (Med), MS, FRACS (Plast), Visiting Medical Officer, Department of Plastic Surgery, Sydney Children's Hospital, Randwick, NSW Competing interests: None.

Funding: None.

Provenance and peer review: Not commissioned, externally peer reviewed.

\section{Correspondence to:}

rtshiladze@hotmail.com

\section{References}

1. Johnson D, Wilkie AO. Craniosynostosis. Eur J Hum Genet 2011;19(4):369-76. doi: 10.1038/ ejhg.2010.235.

2. Governale LS. Craniosynostosis. Pediatr Neurol 2015;53(5):394-401. doi: 10.1016/j. pediatrneurol.2015.07.006.

3. Garza RM, Khosla RK. Nonsyndromic craniosynostosis. Semin Plast Surg 2012;26(2):53-63. doi: 10.1055/s-0032-1320063.
4. Kolar JC. An epidemiological study of nonsyndromal craniosynostoses. J Craniofac Surg 2011;22(1):47-49. doi: 10.1097/ SCS.0b013e3181f6c2fb.

5. Di Rocco F, Arnaud E, Renier D. Evolution in the frequency of nonsyndromic craniosynostosis. J Neurosurg Pediatr 2009;4(1):21-25. doi: 10.3171/2009.3.PEDS08355.

6. Panigrahi I. Craniosynostosis genetics: The mystery unfolds. Indian J Hum Genet 2011;17(2):48-53. doi: 10.4103/0971-6866.86171.

7. Rogers GF. Deformational plagiocephaly, brachycephaly, and scaphocephaly. Part I: terminology, diagnosis, and etiopathogenesis. J Craniofac Surg 2011;22(1):9-16. doi: 10.1097/ SCS.0b013e3181f6c313.

8. Linz C, Kunz F, Böhm H, Schweitzer T. Positional skull deformities. Dtsch Arztebl Int 2017;114(31-32):535-42. doi: 10.3238/ arztebl.2017.0535.

9. Kajdic N, Spazzapan P, Velnar T. Craniosynostosis Recognition, clinical characteristics, and treatment. Bosn J Basic Med Sci 2018;18(2):110-16 doi: 10.17305/bjbms.2017.2083.

10. Tahiri Y, Bartlett SP, Gilardino MS. Evidence-based medicine: Nonsyndromic craniosynostosis. Plast Reconstr Surg 2017;140(1):177e-91e. doi: 10.1097/ PRS.0000000000003473.

11. Fearon JA, Singh DJ, Beals SP, Yu JC. The diagnosis and treatment of single-sutural synostoses: Are computed tomographic scans necessary? Plast Reconstr Surg 2007;120(5):1327-31. doi: 10.1097/01. prs.0000279477.56044.55.

12. van Wijk RM, van Vlimmeren $L A$, Groothuis-Oudshoorn CG, Van der Ploeg CP, ljzerman MJ, Boere-Boonekamp MM. Helmet therapy in infants with positional skull deformation: Randomised controlled trial. BMJ 2014;348:g2741. doi: 10.1136/bmj.g2741.

13. Fearon JA, Ditthakasem K, Herbert M, Kolar J. An appraisal of the cephalic index in sagittal craniosynostosis, and the unseen third dimension. Plast Reconstr Surg 2017;140(1):138-45. doi: 10.1097/PRS.0000000000003422.

14. Ferreira MP, Collares MV, Ferreira NP, Kraemer JL, Pereira Filho Ade A, Pereira Filho Gde A. Early surgical treatment of nonsyndromic craniosynostosis. Surg Neurol 2006;65 Suppl 1:S22-26. doi: 10.1016/j.surneu.2005.11.038.

15. Chim H, Gosain AK. An evidence-based approach to craniosynostosis. Plast Reconstr Surg 2011;127(2):910-17. doi: 10.1097/ PRS.0b013e318204649a.

16. Alperovich M, Vyas RM, Staffenberg DA. Is craniosynostosis repair keeping up with the times? Results from the largest national survey on craniosynostosis. J Craniofac Surg 2015;26(6):1909-13. doi: 10.1097/ SCS.0000000000001300.

17. Al-Shaqsi S, Zellner E, Ching J, Forrest C, Phillips J. The natural history of cranial morphology in sagittal craniosynostosis. J Craniofac Surg 2018;29(4):852-55. doi: 10.1097/ SCS.0000000000004298.

18. Ozgur BM, Aryan HE, Ibrahim D, et al. Emotional and psychological impact of delayed craniosynostosis repair. Childs Nerv Syst 2006;22(12):1619-23. doi: 10.1007/s00381-0060148-x.

19. Brown L, Proctor MR. Endoscopically assisted correction of sagittal craniosynostosis. AORN J 2011;93(5):566-79. doi: 10.1016/j.aorn.2010.11.035.
20. Doumit GD, Papay FA, Moores N, Zins JE. Management of sagittal synostosis: A solution to equipoise. J Craniofac Surg 2014;25(4):1260-65. doi: 10.1097/SCS.0b013e3182a24635.

21. Thomas GP, Johnson D, Byren JC, et al. The incidence of raised intracranial pressure in nonsyndromic sagittal craniosynostosis following primary surgery. J Neurosurg Pediatr 2015;15(4):350-60. doi: 10.3171/2014.11.PEDS1426.

22. Taylor WJ, Hayward RD, Lasjaunias P, et al. Enigma of raised intracranial pressure in patients with complex craniosynostosis: The role of abnormal intracranial venous drainage. J Neurosurg 2001;94(3):377-85. doi: 10.3171/ jns.2001.94.3.0377.

23. Gonsalez S, Hayward R, Jones B, Lane R. Upper airway obstruction and raised intracranial pressure in children with craniosynostosis. Eur Respir J 1997;10(2):367-75. doi: 10.1183/09031936.97.10020367.

24. Panchal J, Uttchin V. Management of craniosynostosis. Plast Reconstr Surg 2003;111(6):2032-48. doi: 10.1097/01. PRS.0000056839.94034.47.

25. Persing JA. MOC-PS(SM) CME article: Management considerations in the treatment of craniosynostosis. Plast Reconstr Surg 2008;121 Suppl 4:S1-11. doi: 10.1097/01. prs.0000305929.40363.bf.

26. Utria AF, Mundinger GS, Bellamy JL, et al. The importance of timing in optimizing cranial vault remodeling in syndromic craniosynostosis. Plast Reconstr Surg 2015;135(4):1077-84. doi: 10.1097/ PRS.0000000000001058.

27. Safran T, Viezel-Mathieu A, Beland B, Azzi AJ, Galli R, Gilardino M. The state of technology in craniosynostosis. J Craniofac Surg 2018;29(4):904-07. doi: 10.1097/ SCS.0000000000004399.

28. Ho OA, Saber N, Stephens D, et al. Comparing the use of $3 \mathrm{D}$ photogrammetry and computed tomography in assessing the severity of single-suture nonsyndromic craniosynostosis. Plast Surg (Oakv) 2017;25(2):78-83. doi: 10.1177/2292550317694845.

29. Wilbrand JF, Szczukowski A, Blecher JC, et al. Objectification of cranial vault correction for craniosynostosis by threedimensional photography. J Craniomaxillofac Surg 2012;40(8):726-30. doi: 10.1016/j. jcms.2012.01.007.

30. Barbero-García I, Lerma JL, Marqués-Mateu Á, Miranda P. Low-cost smartphone-based photogrammetry for the analysis of cranial deformation in infants. World Neurosurg 2017;102:545-54. doi: 10.1016/j.wneu.2017.03.015.

correspondence ajgp@racgp.org.au 